\title{
Peripheral neuroimmune interactions: selected review and some clinical implications
}

\author{
Kamal Shouman $^{1}$ (D) Eduardo E. Benarroch ${ }^{1}$
}

Received: 14 December 2020 / Accepted: 9 February 2021 / Published online: 28 February 2021

๑) Springer-Verlag GmbH Germany, part of Springer Nature 2021

\begin{abstract}
Purpose To provide a brief and focused review on peripheral neuroimmune interactions and their implications for some clinical disorders.

Methods Narrative review of the literature including of English-language articles published between 1985 and 2021 using PubMed and MEDLINE.

Results Many studies on experimental models and in vitro indicate that there are close interactions between the neural and immune systems. Processes from sensory afferents and autonomic efferents co-localize with immune cells and interact at discrete anatomical sites forming neuroimmune units. These neuroimmune interactions are bidirectional and mediated by a wide range of soluble factors including neuropeptides, classical neurotransmitters, cytokines, and other molecules that mediate complex cross-talk among nerves and immune cells. Small-diameter sensory afferents express a wide range of receptors that respond directly to tissue damage or pathogen signals and to chemokines, cytokines, or other molecules released from immune cells. Reciprocally, immune cells respond to neurotransmitters released from nociceptive and autonomic fibers. Neuroimmune interactions operate both at peripheral tissues and at the level of the central nervous system. Both centrally and peripherally, glial cells have a major active role in this bidirectional communication.

Conclusions Peripheral neuroimmune interactions are complex and importantly contribute to the pathophysiology of several disorders, including skin, respiratory, and intestinal inflammatory disorders typically associated with pain and altered barrier function. These interactions may be relevant for persistence of symptoms in disorders associated with intense immune activation.
\end{abstract}

Keywords Neuroimmune $\cdot$ Nociceptors $\cdot$ Sympathetic $\cdot$ Cholinergic

$\begin{array}{ll}\text { Abbreviations } \\ \text { AR } & \text { Adrenoceptor } \\ \text { ATP } & \text { Adenosine triphosphate } \\ \text { CCL2 } & \text { Chemokine (C-C motif) ligand 2 } \\ \text { CXCL1 } & \text { C-X-C chemokine ligand 1 (CXCL1) } \\ \text { CGRP } & \text { Calcitonin-gene related peptide } \\ \text { CRPS } & \text { Chronic regional pain syndrome } \\ \text { CSF-1 } & \text { Colony stimulating factor 1 } \\ \text { ENS } & \text { Enteric nervous system } \\ \text { ET-1 } & \text { Endothelin 1 } \\ \text { HSC } & \text { Hematopoietic stem cells } \\ \text { IL } & \text { Interleukin } \\ \text { ILC2 } & \text { Innate lymphoid cell } 2\end{array}$

Eduardo E. Benarroch

benarroch.eduardo@mayo.edu

1 Department of Neurology, Mayo Clinic, 200 First Street SW, Rochester, MN 55905, USA

$\begin{array}{ll}\text { M } & \text { Muscarinic } \\ \text { nAChR } & \text { Nicotinic acetylcholine receptors } \\ \text { NGF } & \text { Nerve growth factor } \\ \text { NK } & \text { Natural killer cell } \\ \text { PGE2 } & \text { Prostaglandin E2 } \\ \text { ROS } & \text { Reactive oxygen species } \\ \text { Th } & \text { T helper cell } \\ \text { TLR } & \text { Toll-like receptor } \\ \text { TNF- } \alpha & \text { Tumor necrosis factor- } \alpha \\ \text { TRPA1 } & \text { Transient receptor potential ankyrin type 1 } \\ \text { TRPV1 } & \text { Transient receptor potential vanilloid type 1 } \\ \text { Treg } & \text { Regulatory T cells } \\ \text { VIP } & \text { Vasoactive intestinal polypeptide }\end{array}$

M Muscarinic

nAChR Nicotinic acetylcholine receptors

NGF Nerve growth factor

NK Natural killer cell

PGE2 Prostaglandin E2

ROS Reactive oxygen species

Th T helper cell

TLR Toll-like receptor

TNF- $\alpha$ Tumor necrosis factor- $\alpha$

TRPA1 Transient receptor potential ankyrin type 1

TRPV1 Transient receptor potential vanilloid type 1

$\begin{array}{ll}\text { Treg } & \text { Regulatory T cells } \\ \text { VIP } & \text { Vasoactive intestinal polypeptide }\end{array}$ 


\section{Introduction}

Since the pioneering studies of Felten and Felten [1] almost 40 years ago, there has been an increasing awareness of and interest in the interactions between the nervous and the immune systems. At the time of writing this review at the end of January 2021, the term "neuroimmune" yielded more than 3249 entries in PubMed. This emphasizes the fundamental importance of these neuroimmune interactions not only in the maintenance of tissue homeostasis and coordination of host defense against environmental threats, but also in disorders ranging from multiple sclerosis and depression to hypertension and irritable bowel syndrome. The integration between the immune and the nervous systems occur at multiple levels, including peripheral barriers such as the skin and mucosal surfaces, lymphoid organs, and central nervous system (CNS). These interactions are mediated by many chemical signals and involve sensory afferents and autonomic efferents that not only coordinate responses suited to preserve tissue integrity but also may initiate or maintain abnormal function (Fig. 1). The COVID-19 epidemic provides yet another reason to focus attention on these neuroimmune interactions. SARS-CoV-2 infections trigger marked immune activation, which via interactions with visceral afferents, central nervous system, and autonomic efferents provide a mechanistic basis for long-standing symptoms such as fatigue, shortness of breath, chronic cough, nausea, gastrointestinal dysmotility, and postural and exercise intolerance (with or without excessive tachycardia) that affect COVID-19 "long-haulers" [2]. There are several comprehensive reviews on neuroimmune interaction in both normal and pathological conditions [1, 3, 8-10], including the relevance of these interactions at the level of the CNS in the setting of COVID-19 [11]. The purpose of this review is not to provide yet another comprehensive account of the mechanisms of interactions among immune cells, visceral afferents, and autonomic efferents, but rather to emphasize some signaling mechanisms involved in peripheral neuroimmune interactions that may be of potential clinical relevance to clinicians for pathophysiological interpretation and eventual rational management of patients with a wide range of medical disorders. Only some representative examples will be provided here. From the point of view of the authors, an insight into the complexity of peripheral neuroimmune interactions is necessary to avoid oversimplification in management of these patients, particularly in the setting of development of chronic disease.

\section{Innervation of the skin and mucosal surfaces}

Barrier tissues such as the skin, cornea, and mucosa of the respiratory and gastrointestinal tract interface with the environment are highly innervated by sensory afferents and autonomic efferents that participate in complex neuroimmune interactions that allow these tissues to respond adequately to a wide range of environmental stimuli to maintain tissue homeostasis. Most afferents are small-diameter afferents, including small myelinated (Aס) and unmyelinated (C) fibers from nociceptors and visceral receptors that express multiple receptors, including cytokine and danger molecular pattern receptors that respond to chemical signals from immune cells or pathogens $[6,7]$. However, some large $A \beta$ afferents may also respond to such signals and initiate pain [12]. In the skin, these afferents innervate both the dermal and epidermal layers. Small-diameter afferents extend free nerve endings ensheathed by specialized Schwann cells and terminate in close proximity to keratinocytes, fibroblasts, endothelial cells, and resident tissue immune cells [6]. The autonomic innervation of the skin is largely sympathetic and restricted to the dermal layer, targeting blood vessels, lymphatics, apocrine and eccrine glands, and arrector pili muscles [13]. The respiratory and gastrointestinal mucosa maintain an epithelial barrier for commensals, pathogens, and foreign antigens. They harbor a large set of lymphoid tissues and immune cells that interact with neural elements forming neuroimmune cell units [8]. Sensory afferents in these tissues detect local mechanical and chemical stimuli including irritants and signals from the microbiota. The gastrointestinal tract also harbors local neurons in small ganglia forming the enteric nervous system (ENS) and local glial cells. Sympathetic and parasympathetic terminals indirectly control motility and secretion via ENS enteric neurons and affect local immune responses.

\section{Sensory afferents}

Sensory afferents involved in neuroimmune interactions have their cell bodies in the dorsal root ganglia (DRG) and trigeminal ganglion, which innervate the skin, joints, muscle, and viscera, and in the nodose ganglion of the vagus nerve, which provides afferents to visceral organs. DRG afferents project to the dorsal horn; vagal afferents project to the nucleus of the solitary tract in the medulla. The main excitatory neurotransmitter of sensory afferents is l-glutamate. A substantial proportion of nociceptive and visceral afferents also utilize neuropeptides such as substance P, calcitonin gene-related peptide (CGRP) and vasoactive intestinal polypeptide (VIP), as well as adenosine 


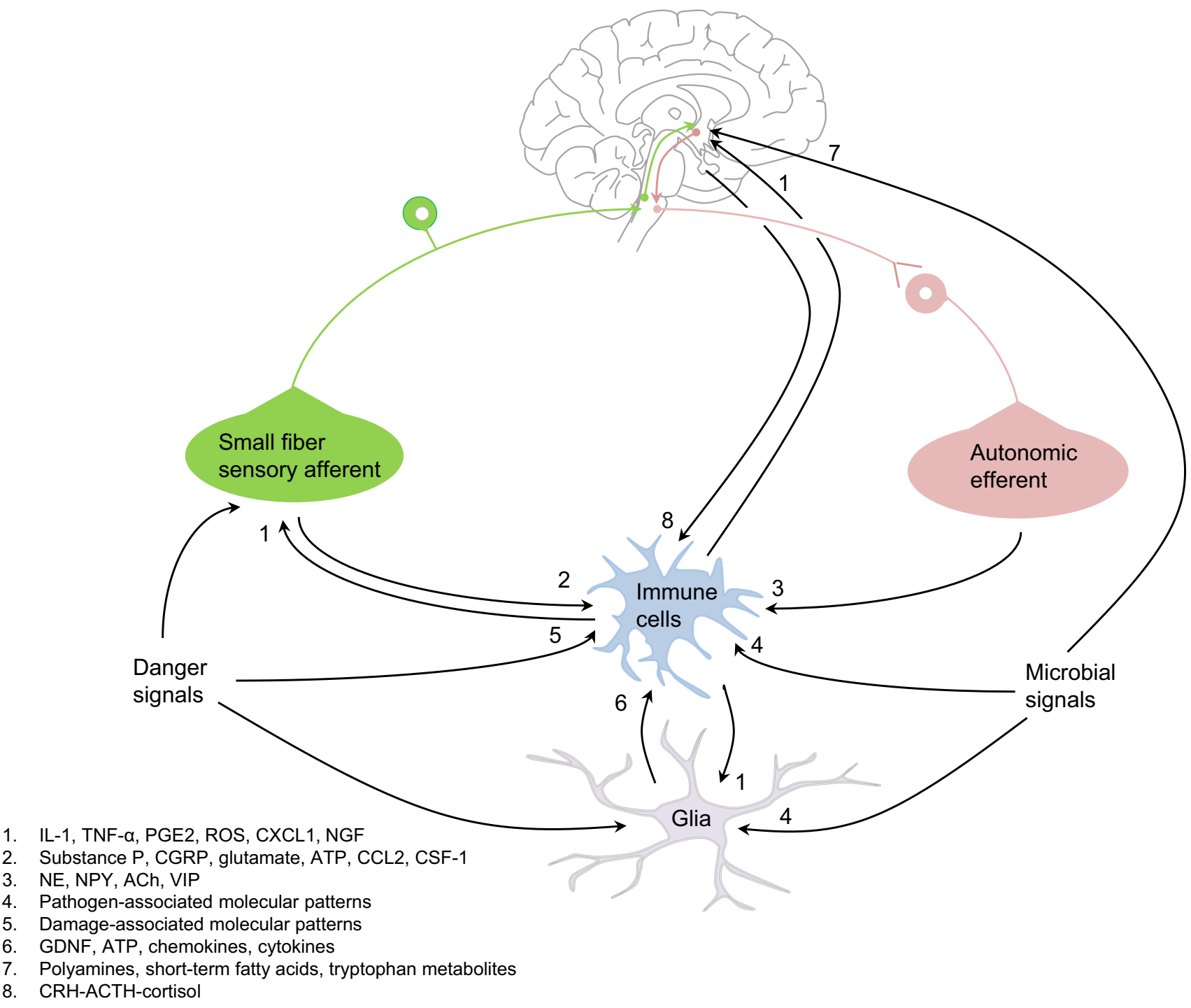

Fig. 1 Reciprocal neuroimmune interactions and some of the major signals involved. Several signals from immune cells, including interleukin (IL)-1, tumor necrosis factor (TNF)- $\alpha$, prostaglandin E2 (PGE2), C-X-C chemokine ligand 1 (CXCL1), and nerve growth factor (NGF) among many others, activate peptidergic small fiber sensory afferents. These afferents release substance $\mathrm{P}$, calcitonin gene related peptide (CGRP), glutamate, adenosine triphosphate (ATP), chemokine (C-C motif) ligand 2 (CCL2) and colony stimulating factor 1 (CSF-1) that activate macrophages and other immune cells. Both primary afferents and immune cells respond to pathogen associated and damage associated molecular patterns. In response to inputs from sensory afferents and circulating cytokines, the nervous system

triphosphate (ATP), and other neurotransmitters. These afferents express a unique repertoire of receptors and ion channels that eventually lead to their depolarization via voltage-gated sodium channels, primarily $\mathrm{Na}_{\mathrm{v}} 1.7$, $\mathrm{Na}_{\mathrm{v}} 1.8$, and $\mathrm{Na}_{\mathrm{v}} 1.9$. A major feature of these afferents is the expression of calcium-permeable transient receptor potential (TRP) channels including vanilloid type 1 activates autonomic outputs that modulate immune cells, including sympathetic outputs utilizing norepinephrine (NE) and neuropeptide $\mathrm{Y}$ or parasympathetic outputs mediated by acetylcholine (ACh), vasoactive intestinal polypeptide (VIP) and other signals. The brain also regulates immune responses via the hypothalamus-pituitary adrenocortical axis involving corticotrophin releasing hormone $(\mathrm{CRH})$ and corticotrophin (ACTH) leading to release of cortisol. Local glial cells participate in neuroimmune interactions. For example, enteric glia release glial-derived neurotrophic factor (GDNF) that activates both neurons and glial cells. In addition to pathogen associated signals, the microbiota may release other signals that may reach the brain and modulate neuroinflammatory responses

(TRPV1) activated by noxious heat and acid, and ankyrin type 1 (TRPA1) channels activated by reactive oxygen species (ROS). They also express G-protein-coupled receptors including histamine, bradykinin, prostaglandin E2 (PGE2), sphingosine-1 phosphate, prokineticin, purinergic $\mathrm{P} 2 \mathrm{Y}$, chemokine, and the Mas-related family of G-proteincoupled receptors (Mrgpr), as well as purinergic P2X and 
serotonergic 5-HT3 receptors and acid-sensing cation channels, hyperpolarization-activated cyclic nucleotidegated channels, and anoctamin 1 channels $[14,15]$. Relevant for neuroimmune interactions, small fiber afferents express receptors for chemokine (C-C motif) ligand 2 (CCL2) and C-X-C chemokine ligand 1 (CXCL1); for proinflammatory cytokines such as interleukin- $1 \beta$ (IL-1 $\beta$ ) and tumor necrosis factor- $\alpha$ (TNF- $\alpha$ ); for damage/pathogenassociated molecular patterns including Toll-like receptors (TLRs) and nucleotide-binding oligomerization domain (NOD)-like receptors; and for nerve growth factor (NGF) $[16,17]$. These signals elicit nociceptor sensitization both by increasing transcription of $\mathrm{Na}_{\mathrm{v}}$ and TRP channels and via post-transcriptional mechanisms that directly promote gating of these channels. Vagal afferents innervating the liver and other visceral organs have a major role in neuroimmune interactions [18]. Upon depolarization, peptidergic DRG and vagal sensory afferents release glutamate, substance P, CGRP, VIP and other signals at both their central and peripheral terminals. Central projections activate neurons in the dorsal horn or nucleus of the solitary tract (NTS) that initiate autonomic reflexes that affect peripheral immune responses. For example, hepatic vagal sensory afferent nerves indirectly sense the gut microenvironment and relay these inputs to the NTS, which via the dorsal vagal parasympathetic output and enteric neurons control immune responses in the gut [19]. Sensory afferents, via the dorsal horn and NTS also relay immune signals to the upper brainstem, hypothalamus, amygdala and thalamic relay nuclei projecting to the insular cortex. Circulating cytokines may have direct access to the hypothalamus and medulla via circumventricular organs that lack a blood brain barrier or through paracrine signaling from brain endothelial cells, for example via PGE2 [20]. Neurons in the hypothalamus and medulla initiate homeostatic and survival responses, including immunomodulation, via descending autonomic (primarily sympathetic) output and endocrine signals, primarily the hypothalamicpituitary-adrenocortical axis [7,21].

\section{Sympathetic efferents}

The sympathetic output originates from preganglionic neurons located in the intermediolateral cell column of the thoracolumbar spinal cord. These neurons receive inputs from segmental efferents and descending projections from the brainstem and hypothalamus and provide cholinergic excitatory input to the sympathetic ganglia and adrenal medulla via ganglion-type nicotinic acetylcholine receptors (nAChRs) [22]. There is abundant sympathetic innervation of lymphoid organs [4, 9, 23, 24]. Paravertebral ganglia innervate the blood vessels and sweat glands of the face, trunk and limbs, and the bone marrow and thymus.
Prevertebral ganglia innervate abdominal and pelvic viscera as well as the spleen and the gut lymphoid system. The primary neurotransmitter in sympathetic efferents is norepinephrine, which acts via several types of $\alpha$ - and $\beta$-adrenergic receptors (adrenoceptors, ARs). Some sympathetic noradrenergic efferents also release neuropeptide Y (NPY) and other signals that may also affect immune cells [1].

\section{Parasympathetic efferents}

The parasympathetic innervation of the viscera originates from local and intramural ganglia that receive excitatory cholinergic input from preganglionic neurons located in the brainstem and sacral spinal cord. Preganglionic input to thoracic and abdominal ganglia originates from the dorsal motor nucleus of the vagus, whereas that to pelvic ganglia originates in the sacral parasympathetic nucleus. Local ganglia, including those located in the heart, respiratory tract and ENS contain complex circuits that integrate local, parasympathetic preganglionic, and sympathetic influences. The primary neurotransmitter of most parasympathetic ganglia and ENS is ACh, which mediates excitatory responses such as smooth muscle contraction and glandular secretion via M3 muscarinic receptors. In contrast, vagal inputs to the heart reduce automatism and conduction via inhibitory M2 receptors. Smooth muscle relaxation and vasodilation triggered by the parasympathetic system are mediated by noncholinergic neurons that utilize nitric oxide (NO), VIP, and other signals. Cholinergic signals control immune responses via muscarinic receptors. For example, activation of muscarinic acetylcholine receptors directly induced aldehyde dehydrogenase gene expression in both human and mouse colonic antigen-presenting cells promoting peripheral $\mathrm{T}$ regulatory cell (Treg) proliferation [19].

\section{Immune cells as triggers and targets of neuroimmune interactions}

Immune cells both release signals that activate nerve afferents and are a target of signals from sensory afferents and autonomic efferents. A comprehensive discussion on the different types and function of immune cells is beyond the scope of this review, and only a few salient aspects will be emphasized here. Immune cells originate from hematopoietic stem cells (HSCs) that give rise to all blood cell lineages [25]. Hematopoiesis in adults mostly takes place in the bone marrow, whereas T-lymphocyte development occurs in the thymus [26]. Innate lymphoid cells (ILCs) are in both tissues and peripheral blood and encompass several populations, including cytotoxic ILCs (NK cells), helper ILCs and regulatory ILCs [27]. Helper ILCs, including ILC2 cells that reside in close proximity to neurons and glial cells at mucosal 
barriers, drive immunological responses via production of interferon (IFN)- $\gamma$, tumor necrosis factor (TNF)- $\alpha$, interleukin (IL)-13 and IL-17A [28]. For example, ILC2 expansion and production of IL-13 and IL-5 in the context of allergic disease explain tissue eosinophilia, increased mucus production and airway hyper-reactivity; ILC3s exert antimicrobial effects and promote tissue repair and remodeling in the gut [27]. Dendritic cells are innate immune cells that provide an essential interface between the innate and adaptive immunity. They are found in blood, tissues and lymphoid organs, recognize and respond to pathogen-associated and danger-associated signals, and shape the acute inflammatory response [29]. They process extracellular and intracellular proteins and present antigens in the context of major histocompatibility complex (MHC) molecules to prime naive $\mathrm{T}$ cells. There are several different types of dendritic cells, each specialized to respond to particular pathogens and to interact with specific subsets of $\mathrm{T}$ cells and, depending on the context, can either promote T-cell activation or induce tolerance $[29,30]$. Tissue-resident macrophages constitute a highly heterogeneous population found in most body tissues [31, 32]. Macrophages exhibit different functional states and have been classically subdivided into two major phenotypes. M1-like macrophages are polarized by signals such as bacterial lipopolysaccharides or Thelper (Th) 1 cytokines such as IFN- $\gamma$ and secrete pro-inflammatory cytokines such as IL-1 $\beta$, IL-6, IL-12, IL-23, and TNF- $\alpha$; M2-like macrophages are polarized by Th 2 cytokines such as IL-4 and IL-13, secrete anti-inflammatory cytokines such as IL-10 and transforming growth factor (TGF)- $\beta$, and contribute to tissue repair [33]. However, there are many intermediate phenotypes that vary according to the environmental stimuli.

T-lymphocyte activation is a complex process [10]. The canonical mechanism depends on the T-cell receptor (TCR)MHC interaction that triggers a downstream cascades that eventually result in activation of nuclear factor kappa-lightchain enhancer of activated B cells (NF- $\mathrm{KB}$ ), which promotes transcription of genes crucial to activation and proliferation. In addition, changes in intracellular $\mathrm{Ca}^{2+}$ results in activation and nuclear translocation of nuclear factor of activated $\mathrm{T}$ cells, which controls transcription of IL-2, the principal T-cell growth factor. $\mathrm{T}$ lymphocytes are primarily categorized by their expression of cluster of differentiation (CD) markers; the segregation between $\mathrm{CD} 4^{+}$and $\mathrm{CD} 8^{+} \mathrm{T}$ lymphocytes occurs during their selection in the thymus. CD4+ T lymphocytes communicate with MHC II in antigen presenting cells and respond to cytokine signals further differentiating into effector cell subtypes including $\mathrm{T}$ helper type 1 (Th1), T helper type 2 (Th2) cells, or T helper type 17 (Th17) cells. Secretion of IL-12 by dendritic cells results in polarization to Th 1 cells, which secrete IL-2, IFN- $\gamma$, and TNF- $\alpha$ secretion favoring classical cell-mediated immunity. The absence of IL-12 and presence of IL-4 favors the Th2 subtype, which activates B-lymphocytes and result in isotype switching to immunoglobulin $\mathrm{E}$ and eosinophil activation. Secretion of IL-23, TGF- $\beta$, and IL- 6 by dendritic cells results in polarization to Th17 subtype, which secretes IL-17 and has a major role in autoimmune diseases. In addition to polarization to effector subtypes, CD4+ T lymphocytes can also differentiate to regulatory $\mathrm{T}$ lymphocytes (Tregs), which secrete IL-10, resulting in the suppression of antigen-specific effector T lymphocytes. CD8+ T lymphocytes interact with MHC I presented by all nucleated cells and release both cytotoxic granules that can damage membranes and activate apoptosis and proinflammatory cytokines such as IFN- $\gamma$.

B cells are also a crucial component of the adaptive immune system; some ultimately differentiate to antibody secreting plasma cells. In autoimmune disorders, B lymphocytes may also function as antigen-presenting cells, producing proinflammatory cytokines that induce Th1 and Th17 responses [34].

Of note, many types of immune cells produce neurotransmitters that can act in a paracrine function modulating immune responses. For example, many immune cells produce neuromodulatory neuropeptides, including substance $\mathrm{P}$, CGRP, and vasoactive intestinal polypeptide, among many others [35]. T lymphocytes can synthesize both catecholamines and acetylcholine and regulate their production; these signals are important where there does not appear to be a clear anatomical neuroimmune connection, such as the parasympathetic nervous system communicating with splenic $\mathrm{T}$ -lymphocytes [10].

In addition to myeloid cells mediating innate immunity and lymphocytes mediating adaptive immunity, endothelial cells, epithelial cells, and fibroblasts express genes involved in immune responses and may potentially participate in neuroimmune interactions critical for organ-specific homeostasis [36].

\section{Signals from immune cells to nociceptors}

Immune cells produce several mediators that activate sensory neurons. For example, macrophages express different TLRs (e.g. TLR4) that trigger expression of proinflammatory cytokines and chemokines that have major role in triggering neuropathic pain [16, 17, 37]. Upon activation after tissue injury macrophages release CXCL1, IL-1 $\beta$, IL-6, TNF- $\alpha$, PGE2, ROS, NGF and other signals that activate nociceptors and promote nociceptor sensitization [16]. In addition, at the site of peripheral nerve injury, endothelial activation may result in recruitment of $\mathrm{CX} 3 \mathrm{C}$ chemokine receptor 1 (CX3CR1)-expressing macrophages, which release ROS that sensitize nociceptive afferents via TRPA1 channels [37]. Complement C5a may activate C5aR1 in skin macrophages, promoting macrophage-dependent signaling that involves mobilization of NGF and sensitization 
of TRPV1 expressing nociceptors [38]. In contrast, macrophages with M2-like phenotype may have an active role in the resolution of pain by releasing anti-inflammatory mediators such as IL-10 and transforming growth factor- $\beta$ (TGF- $\beta$ ) and pro-resolving mediators such as neuroprotectin D1 [16].

\section{Detection of danger signals by nociceptors}

Like immune cells, nociceptive neurons respond to damage/pathogen -associated molecular patterns through pattern recognition receptors, including TLRs and NOD-like receptors that lead to the formation of the NLRP3 inflammasome with cleavage and activation of pro-IL- $1 \alpha$ and pro-IL-18 [17]. Thus, pathogen- or danger-associated molecular patterns can activate nociceptive neurons both via an indirect pathway mediated by signals from immune cells and via direct activation of TLRs expressed by nociceptors. In addition to slow-acting canonical TLR signaling in nociceptors, TLRs also couple to ion channels in sensory neurons, enabling rapid modulation of neuronal activity. For example, TLR4 (and TLR3) may directly activate TRPV1 and TRPA1 channels in nociceptors. Activation of sphingosine-1 receptors may also sensitize nociceptors both via TRPV1 and TRPA1 channels and through direct action of ion potassium and chloride channels. Nociceptors also rapidly respond to microbial signals [39]. For example, bacteria may induce calcium flux and depolarization in nociceptor neurons, in part via bacterial $N$-formyl peptides and the pore-forming toxin alpha-hemolysin [40]. Bacterial lipopolysaccharides directly activate TLR4 in nociceptors leading to sensitization via TRPV1 [41] or TRPA1 [42] channels. Endothelial signals may also trigger nociceptor sensitization. For example, activation of the mechanically-gated ion channel Piezo2 in endothelial cells leads to release of the pro-nociceptive vasodilator endothelin-1 (ET-1), which elicits nociceptor sensitization [43]; ET-1 may also promote endothelial cell release of ATP in response to mechanical stimulation leading to activation of $\mathrm{P} 2 \mathrm{X} 2 / 3$ receptors in nociceptors [44].

\section{Activating signals from nociceptors to immune cells}

In response to large variety of stimuli in peripheral tissues, peptidergic sensory afferents function as "sensorimotor nerves" that release substance P, CGRP, ATP, brain-derived neurotrophic factor, and other signals antidromically at the site of injury. Substance P and CGRP act via receptors on blood vessels and immune cells, eliciting "neurogenic inflammation" [45-47].

This response manifests with nociceptor sensitization, endothelium-dependent vasodilation, mast cell degranulation, edema, local activation of dendritic cells, macrophages, and $\mathrm{T}$ cells, and immune cell extravasation. The sensory ganglia, including the DRG, trigeminal ganglion, and nodose ganglion host many different types of immune cells, which allows for local neuroimmune interactions relevant for neuropathic pain $[48,49]$. Satellite glia in the DRG participate in cross-talk with macrophages promoting nociceptor sensitization [50,51]. Nerve injury triggers upregulation of membrane TLR7 in peptidergic nociceptive neurons leading to release of CCL2, IL-1 $\beta$, TNF- $\alpha$ and extracellular vesicles containing microRNAs (mRNAs) that trigger macrophage activation [48]. For example, paclitaxel activates TLR4 signaling in nociceptors leading to increased expression of CCL2 by DRG neurons and local macrophage infiltration, promoting neuropathic pain [52]. Nociceptor-derived microRNA (miR)-21 containing vesicles are engulfed to macrophages and polarizes these cells towards a pro-inflammatory/pronociceptive phenotype $[37,53]$. After nerve injury CCL2, and colony stimulating factor 1 (CSF-1) are induced in DRG neurons and transported to axonal terminals in the dorsal horn, where they promote microglial activation [54]. Microglia-astrocyte interactions in the dorsal horn has an important role in pain central sensitization [55]. In response to injury or inflammation, prokineticin 2 signaling is upregulated in DRG neurons, leading both to tonic activation of TRPV1 and TRPA1 contributing to peripheral sensitization and dorsal horn signaling promoting astrocytic activation and central sensitization [56].

\section{Modulatory signals from primary afferents to immune cells}

Neuropeptides released from primary participate in immune modulation by several mechanisms. For example, substance $\mathrm{P}$ acting via neurokinin 1 receptors has a positive stimulatory effect on HSC activity and hematopoiesis [57], sustains the survival of activated T cells [58], stimulates macrophage production of proinflammatory mediators, and primes neutrophils to chemotaxis and migration [39]. In, contrast, CGRP acts as a negative regulator of innate immune responses and contributes to limiting tissue damage in inflammatory disorders. CGRP directly inhibits the ability of macrophages and dendritic cells to produce inflammatory cytokines and to present antigens to T cells [59]. Effector mechanisms include upregulation of IL-10 and inhibition of NF- $\mathrm{kB}$ activity and are triggered by cyclic AMP-protein kinase, a pathway downstream of its the CGRP receptor activation. CGRP also reduces monocyte recruitment and macrophage killing ability and inhibits ILC2 responses [60]. In a murine model of acute lung injury induced by lipopolysaccharide, CGRP attenuated inflammation by regulating macrophage polarization [61]. This effect involved reduced NLRP3 and pro-IL- $1 \beta$ mRNA expression and enhanced the IL-4 induced expression of markers of M2 macrophages (such as IL-10). These effects of CGRP 
involved a transduction pathway that included calmodulin, protein kinase $\mathrm{C}$ and protein kinase $\mathrm{A}$ [61].

\section{Effects of autonomic outputs on neuroimmune interactions}

The sympathetic and parasympathetic outputs have a major role in regulating immune responses $[4,9,23]$. Whereas the sympathetic output typically leads to general and patterned modulation of immune responses, parasympathetic outputs typically act in individual organs in response to local signals.

\section{Sympathetic output and modulation of immune responses}

The sympathetic nervous system impacts function, survival, proliferation, circulation, and trafficking of immune cells. Sympathetic fibers provide a robust input to the bone marrow $[62,63]$ as well as lymph nodes, spleen and gut-associated lymphoid tissue, terminating in the vicinity of immune cell clones and stromal cells [4, 23, 24, 64]. In the bone marrow, sympathetic efferents form complexes with stromal cells in hematopoietic niches; catecholamines promote the egress of hematopoietic cells from the bone marrow [65]. This effect is important for the circadian mobilization of HSCs to the bloodstream; circadian oscillations of norepinephrine levels induce rhythmic expression of CXCL12 by mesenchymal stem and progenitor cells leading to the rhythmic release of HSCs from the bone marrow [65]. The sympathetic innervation of the bone marrow declines with age; this may contribute to the decline in regenerative capacity and differentiation potential of HSCs with aging [66]. Sympathetic nerve fibers also terminate in close proximity to thymocytes, thymic epithelial cells, mast cells, and macrophages in the thymus [67], where norepinephrine may inhibit thymopoiesis [68]. Norepinephrine is essential for antigen processing and trafficking of activated lymphocytes into circulation [69, 70]. Several lymphocyte subtypes express cell-intrinsic circadian clocks [71], which may contribute to rhythmicity to lymphocyte migration [72].

Dendritic cells, macrophages and lymphocytes express $\alpha$ ARs and $\beta$ ARs in different proportion and distributions $[10,73,74]$. Adrenoceptor expression varies among the immune cell types and according to the degree of cell activation. Several environmental factors present during inflammation can regulate AR expression on immune cells, including TLR ligands, hormones, and cytokines. Dendritic cells, macrophages and natural killer (NK) cells express both $\alpha \mathrm{ARs}$ and $\beta$ ARs. Expression of $\beta 2 A R$ in T cells differs across the different subsets; among CD4+ cells, $\beta 2$ ARs are expressed in Th1 and regulatory Treg but is absent in Th2 cells due to IL-4-mediated epigenetic modifications. Several in vitro studies indicate that the sympathetic output potently modulates dendritic cells, $\mathrm{T}$ and $\mathrm{B}$ lymphocytes via $\beta 2 \mathrm{AR}$ signaling [30]. Activation of $\beta 2 A R s$ in dendritic cells inhibits antigen cross-presentation to $\mathrm{CD} 8+\mathrm{T}$ cells and dendritic cell release of TNF- $\alpha$, IL-12, and IL-6, while increasing IL-10 and IL-33 levels. Activation of $\beta 2$ ARs also suppresses Th1 immune responses; promotes Th2 and Treg responses; inhibits ICL2 responses; and suppresses circulating NK cell numbers and activity $[75,76]$. In contrast, activation of $\alpha 1 \mathrm{ARs}$ is essential for migration of dendritic cells to lymph nodes and skin where $\alpha 2$ ARs promote antigen uptake by dendritic cells; $\alpha 1$ ARs may also promote cytotoxicity of spleen NK cells. In general, chronic inflammation changes the profile of AR expression on immune cells, inhibiting $\beta A R$ and enhancing $\alpha A R$ expression, which might be related to the pro-inflammatory process in some immune-mediated diseases.

The sympathetic output to the spleen originates in the superior mesenteric and celiac ganglia; postganglionic fibers enter via the splenic nerve and form a dense network in the white pulp in the vicinity of $\mathrm{T}$ cells, macrophages, and B cells [24]. Sympathetic influences on the spleen have a major role in regulation of immune responses and mediate the efferent component of the so-called cholinergic or vagal anti-inflammatory pathway triggered by vagal nerve stimulation several experimental models [77]. The mechanisms of this anti-inflammatory response are still poorly understood [78]. Evidence indicates that the sympathetic and not the vagal outputs are the essential component of the reflex. Vagus nerve stimulation triggers sympathetic output to the spleen, where $\beta 2 \mathrm{AR}$ signaling induces release of acetylcholine from local CD4 $\mathrm{T}$ lymphocytes; this leads to activation of $\alpha 7$ nicotinic acetylcholine receptors (nAChRs) in splenic macrophages, suppressing their production of TNF- $\alpha$ and other proinflammatory cytokines. In the spleen, few cholinergic $\mathrm{T}$ or B cells are located close to adrenergic terminals; these lymphocytes express the chemokine CXCR5 receptor and respond to CXCL13 produced by stromal cells that express abundant $\beta 2$ Ars [79]. This suggests that sympathetic modulation of immune function in the spleen involves chemokine signaling from stromal cells. In this pathway, vagal afferent inputs reach the nucleus of the solitary tract, which via projections to the paraventricular nucleus of the hypothalamus and premotor sympathoexcitatory $\mathrm{C} 1$ neurons of the rostral ventrolateral medulla, triggers potent anti-inflammatory responses via activation of preganglionic neurons of the spinal cord [80]. Neuropeptide Y released from sympathetic adrenergic terminals may also contribute to immune modulation via different receptor subtypes expressed in dendritic cells, macrophages, lymphocytes, and NK cells [81].

Sympathetic nerve neuroplasticity following injury may contribute to dysregulation of secondary lymphoid organs 
[82]. Studies in experimental models show that, whereas immune activation elicits a rapid and selective increase in splenic sympathetic activity in the early phase of the immune response, sympathetic activity in the spleen and lymph nodes decreases thereafter during prolonged acute and chronic immune responses. A postmortem study showed loss of sympathetic nerves in the spleen of patients who died of sepsis compared to those who died from non-inflammatory conditions [83]. This adaptive sympathetic nerve plasticity may reflect axonal retraction and/or segmental axonal degeneration of sympathetic fibers terminals triggered by cytokines, neurotrophins, or semaphorins released from immune or stromal cells [84].

In addition to the systemic immunomodulatory actions, sympathetic output also participates in local neuroimmune interactions that may have disease implications. Sympathetic nerve terminals densely innervate the ENS neurons inhibiting intestinal motility, and also terminate in close proximity to dendritic cells, plasma cells, and T-cell zones in Peyer patches [85]. Sympathetic-ILC2 units mediate a regulatory sympathetic circuit that via $\beta 2 \mathrm{ARs}$ dampens ILC2-mediated type 2 inflammation at mucosal surfaces [85]. Sympathetic activation enhances the tissue-protective M2-phenotype of muscularis macrophages suppressing TNF- $\alpha$ secretion and phagocytosis. Sympathetic inputs acting via $\beta 2 \mathrm{ARs}$ also inhibit the release of histamine and other inflammatory mediators from mast cells in the intestine. Genetic deletion of the Adrb2 gene encoding the $\beta 2 \mathrm{ARs}$ produces increased intestinal inflammation, susceptibility to Listeria monocytogenes infection, and endotoxemia [85].

\section{Parasympathetic interactions with local neurons, immune cells, and glia at mucosal surfaces}

The effects of the parasympathetic outputs on immune responses are complex. Human T lymphocytes express several subtypes of nicotinic and muscarinic receptors. Excitatory muscarinic receptors, particularly M3 receptors, may have an important role in their activation and differentiation. The effects of nicotinic receptors is complex, as they can activate both proinflammatory Th1 and anti-inflammatory Treg cells [10]. T and B cells, macrophages, and dendritic cells express the components necessary for a functional cholinergic transmission as well as several subtypes of muscarinic and nicotinic receptors [86]. Immune activation upregulates the intrinsic cholinergic activity of these cells. Acetylcholine release from cholinergic $\mathrm{T}$ cells in the spleen, and not that released from nerve terminals, is responsible for activation of $\alpha 7 \mathrm{nAChRs}$ in splenic macrophages leading to attenuation of inflammation triggered by vagus nerve stimulation [87]. Vagal efferents interact with multiple cells types at effector organs, influencing local neurons, glial cells, and immune cells, and have thus a major role in controlling barrier permeability. For example, in addition to the "cholinergic anti-inflammatory pathway" centered in the spleen, there is a similar pathway centered in the lung [88]. Vagal cholinergic inputs to the lung activates ILC3 cells producing IL-22, which is a protective mediator of mucosa immunity [88].

The maintenance of gut homeostasis depends on the coordinated development and responses of the ENS and intestinal immune systems. Enteric neurons, glial, and immune cells form neuroimmune units that occupy distinct anatomical niches within the gut and respond to signals from the lumen, including those from the gut microbiota [89]. Vagal activation enhances barrier function and reduces inflammation through $\mathrm{nAChR}$ activation of enteric glia and muscularis macrophages [90]. Enteric neurons maintain muscularis macrophages through secretion of CSF-1, whereas macrophages activate ENS neurons by secreting bone morphogenetic protein 2 [91]. This bidirectional cross-talk regulates the peristaltic activity of the colon and is promoted and activated by commensal microbe-derived signals. Enteric glial cells sense pathogens and produce neurotrophic factors that stimulate protective immune responses that help maintain the epithelial barrier integrity. For example, enteric glia sense microbial and host alarmin cues and release glialderived neurotrophic factor (GDNF) family neurotrophic factors that induce IL-22 production by ILC3s, promoting tissue repair [92]. Members of the GDNF family also activate both ENS development and Peyer patch organogenesis [92].

\section{Clinical correlations}

Evidence from experimental models indicate that neuroimmune interactions contribute to the pathophysiology of skin, respiratory, gastrointestinal, and cardiovascular disorders such as hypertension. These topic has been extensively reviewed $[6,7,10,84]$, and only a few points on some examples will be emphasized here.

\section{Skin disorders}

The interactions between nociceptors on immune cells in the skin are relevant for several inflammatory skin conditions [6, 45-47]. Stress, inflammation, and nerve injury elicit release of substance $P$, which triggers mast cell degranulation by activation of MrgprX2 receptors [93]. Interactions between small sensory afferents and keratinocytes also contribute to inflammatory skin conditions such as atopic dermatitis [94]. This disorder is characterized by chronic pruritus, thickened scaly skin, impaired epidermal barrier function, type $2 \mathrm{~T}$ helper cell-skewed allergic response, and skin hyperinnervation with penetration of sensory afferents 
into the epidermis. In this disorder, keratinocytes trigger itch through their release of histamine, ET-1, and other mediators that activate surrounding pruriceptive small fiber afferents lading to release of substance P and CGRP [95]. These neuropeptides trigger keratinocyte proliferation and release of NGF resulting in increased innervation and leakiness of the skin barrier [96]. Psoriasis is associated with dysregulation in the IL-23/IL-17 axis that may result from interactions among nociceptors, keratinocytes, and local dendritic cells [97]. CGRP released from primary afferents triggers IL-23 production from nearby dermal dendritic cells, which drives IL-17 expression by $\gamma \delta \mathrm{T}$ cells promoting psoriatic inflammation [97]. This pathway also recruits circulating neutrophils and monocytes promoting innate protection against pathogens [98].

\section{Complex regional pain syndrome}

Complex regional pain syndrome (CRPS) is a prototypical example of interactions among nociceptive and sympathetic fibers, immune cells, and glial cells. CRPS is characterized by skin nociceptor sensitization leading to pain and allodynia, time-dependent changes in vasomotor and sudomotor output, inflammation, and CNS plasticity of sensory representation [99-101]. For example, in experimental models of CRPS-1, oxidative stress, activation of macrophage and Schwann cells, and activation of TRPA 1 channels in nociceptors contribute to allodynia [102]. Sympathetic output may contribute to pain in injured or inflamed tissue norepinephrine by several mechanisms, including injury-induced expression of novel $\alpha 1 \mathrm{ARs}$ and changes in ion channel properties on nociceptors leading to sympathetic-nociceptor cross-talk. Norepinephrine acting via $\beta 2 A R s$ in keratinocytes stimulates the production of IL- 6 and leads to nociceptive sensitization [103].

\section{Respiratory allergic and inflammatory disorders}

Epithelial damage during allergic inflammation exposes vagal parasympathetic nerve endings and promotes acetylcholine release. Cholinergic signals acting via M3 muscarinic receptors elicit bronchoconstriction, mucus secretion, and activation of ICL2; activation of this receptor also activates epithelial cells and macrophages, inducing leukotriene B4 release and inflammatory cell chemotaxis. The coordinated activity of vagus nerve endings, airway epithelial cells, pulmonary neuroepithelial cells, and ILC2 cells has a major role in regulating lung infection and immunity, as recently reviewed $[6,90]$. At the mucosal surface, ILC2s are a major effector of neuroimmune interactions triggered by vagal afferents and affecting cytokine production during allergen challenge. Vagal $\mathrm{C}$-afferents both sense and respond to the lung infection and inflammatory cytokines and trigger airway inflammation and resistance by releasing substance $P$, vasoactive intestinal polypeptide, and other mediators. ILC2 cells adjacent to airway epithelial cells express both $\alpha 7 \mathrm{nAChRs}$ and neuropeptide receptors and can thus be regulated by neuropeptides and ACh released from vagal nerve endings. Epithelial-derived cytokines can synergize with neuropeptides and $\mathrm{ACh}$ to boost production of effector cytokines in ILC2 populations and increase airway hypersensitivity under lung viral infection and allergy challenge. Pulmonary neuroendocrine cells are innervated by vagal nerve endings, are in close contact with ILC2 cells, and release CGRP and GABA [90]. These mediators can synergize with epithelium-derived cytokines to facilitate proliferation of ILC2 and production of proinflammatory Th2 cytokines (IL-4, 5 and 13) that potentiate allergic inflammation, as shown in some experimental models of asthma [104]. However, whereas CGRP supports IL-5 production, it constrains ILC2 proliferation [105]. Activation of $\alpha 7 \mathrm{nAChRs}$ by acetylcholine released from vagal afferents also downregulates ICL2-triggered inflammation [106]. Mucosal neurons were found adjacent to ILC2 and directly sense worm products and alarmins to control innate type 2 cytokines [107]. Neuromedin U produced in local mucosal neurons acts via receptors on ILC2 cells promoting production of innate inflammatory and tissue repair cytokines resulting in immediate protective type 2 responses [107]. These complex interactions provide multiple potential pharmacological targets for managements of inflammatory (including allergic) reactions affecting the respiratory tract.

\section{Inflammatory and functional gastrointestinal disorders}

Several gastrointestinal disorders may reflect bidirectional communication between the nervous and immune systems [108, 109]. Abnormal neuroimmune interactions affect barrier function and motility, and in many cases produce abdominal pain. Many of these interactions involve mast cells, which are fundamental elements of the intestinal barrier, as they regulate epithelial function and integrity and modulate both innate and adaptive mucosal immunity. Mast cells contact vagal afferent terminals and express both nAChRs that attenuate mast cell responses and muscarinic receptors that increase mast cell degranulation and histamine release [110]. Histamine can activate myenteric neurons in the small intestine and promote substance $P$ and CGRP release from sensory afferents, leading to visceral hypersensitivity and inflammation. In turn, substance P, CGRP and VIP promote release of histamine from intestinal mast cells. Disruption of the intestinal barrier is associated with increased passage of luminal antigens into the mucosa, which further facilitates mucosal mast cell activation and inflammatory responses [109]. The importance of 
sympathetic-immune interactions for fine-tuning of peristalsis and immune homeostasis is also shown in experimental models of postoperative ileus, colitis, and intestinal infection $[111,112]$. Activation sympathetic output in the setting of stress or in response to inflammatory molecules may lead to impaired gastrointestinal motility as occurs in experimental models of functional dyspepsia [113]. Glialmediated activation of macrophages may also modulate the development of visceral hypersensitivity [114].

\section{Conclusions}

Many studies on experimental models and in vitro indicate that there are close interactions between the neural and immune systems involving multiple signaling mechanisms. Despite the fact that most of these interactions have been shown in vitro and in experimental models, they have relevance to understanding the pathophysiology of clinical disease. However, in humans, the evidence is, so far, largely indirect, as tools utilized to dissect these interactions in experimental studies (e.g., knockout, miRNAs, receptor blocking drugs) are not yet available for clinical use. For example, in patients, drugs such as beta-blockers may affect widespread subpopulations of immune cells with different effects in different tissue and clinical settings. The benefit of vagal nerve stimulation in inflammatory disorders is largely interpreted on the basis of studies in rodent models, but the mechanism is yet to be defined. Whereas this review focused on the interactions at the peripheral level, the CNS has a critical role in mechanisms of neuroimmune communication $[3,5,7$, 9]. These peripheral and central interactions are yet to be fully characterized in vivo and may be influenced by the basal level of neural activity and stage of immune cell differentiation. Nevertheless, this evidence strongly indicates that neuroimmune interactions may be at the core of many, if not most, medical disorders. Disturbance of these interactions both at the periphery and at the level of the CNS not only lead to end-organ dysfunction but may also result in global impairment of neuromodulation and symptoms involving multiple systems. Immune-activated peripheral afferents, circulating cytokines, and microbial products activate neurons and glial cells in the hypothalamus and medulla and trigger sympathetic and humoral responses, such as cortisol release that may contribute to altered homeostasis and persistence of symptoms in disorders such as COVID-19 [11]. The elucidation of the signaling mechanisms involved will likely provide further opportunity for development of rational therapy.

Funding None.

\section{References}

1. Felten DL, Felten SY (1987) Immune interactions with specific neural structures. Brain Behav Immun 1(4):279-283

2. Marshall M (2020) The lasting misery of coronavirus longhaulers. Nature 585(7825):339-341

3. Wrona D (2006) Neural-immune interactions: an integrative view of the bidirectional relationship between the brain and immune systems. J Neuroimmunol 172(1-2):38-58

4. Bellinger DL, Lorton D (2014) Autonomic regulation of cellular immune function. Auton Neurosci 182:15-41

5. Pavlov VA, Chavan SS, Tracey KJ (2018) Molecular and functional neuroscience in immunity. Annu Rev Immunol 36:783-812

6. Blake KJ, Jiang XR, Chiu IM (2019) Neuronal regulation of immunity in the skin and lungs. Trends Neurosci 42(8):537-551

7. Reardon C, Murray K, Lomax AE (2018) Neuroimmune communication in health and disease. Physiol Rev 98(4):2287-2316

8. Godinho-Silva C, Cardoso F, Veiga-Fernandes H (2019) Neuro-immune cell units: a new paradigm in physiology. Annu Rev Immunol 37:19-46

9. Kenney MJ, Ganta CK (2014) Autonomic nervous system and immune system interactions. Compr Physiol 4(3):1177-1200

10. Elkhatib SK, Case AJ (2019) Autonomic regulation of T-lymphocytes: implications in cardiovascular disease. Pharmacol Res 146:104293

11. Goldstein DS (2020) The extended autonomic system, dyshomeostasis, and COVID-19. Clin Auton Res 30(4):299-315

12. Xu ZZ, Kim YH, Bang S, Zhang Y, Berta T, Wang F et al (2015) Inhibition of mechanical allodynia in neuropathic pain by TLR5mediated A-fiber blockade. Nat Med 21(11):1326-1331

13. Gibbons CH, Wang N, Kim JY, Campagnolo M, Freeman R (2020) Skin biopsy in evaluation of autonomic disorders. Continuum (Minneap Minn) 26(1):200-212

14. Khan A, Khan S, Kim YS (2019) Insight into pain modulation: nociceptors sensitization and therapeutic targets. Curr Drug Targets 20(7):775-788

15. Benarroch EE (2015) Ion channels in nociceptors: recent developments. Neurology 84(11):1153-1164

16. Chen O, Donnelly CR, Ji RR (2020) Regulation of pain by neuro-immune interactions between macrophages and nociceptor sensory neurons. Curr Opin Neurobiol 62:17-25

17. Donnelly CR, Chen O, Ji RR (2020) How do sensory neurons sense danger signals? Trends Neurosci 43(10):822-838

18. Berthoud HR, Neuhuber WL (2000) Functional and chemical anatomy of the afferent vagal system. Auton Neurosci 85(1-3):1-17

19. Teratani T, Mikami Y, Nakamoto N, Suzuki T, Harada Y, Okabayashi K et al (2020) The liver-brain-gut neural arc maintains the Treg cell niche in the gut. Nature 585(7826):591-596

20. Garami A, Steiner AA, Romanovsky AA (2018) Fever and hypothermia in systemic inflammation. Handb Clin Neurol 157:565-597

21. Kipnis J (2016) Multifaceted interactions between adaptive immunity and the central nervous system. Science 353(6301):766-771

22. Shouman K, Benarroch EE (2019) Segmental spinal sympathetic machinery: implications for autonomic dysreflexia. Neurology 93(8):339-345

23. Felten DL, Felten SY, Carlson SL, Olschowka JA, Livnat S (1985) Noradrenergic and peptidergic innervation of lymphoid tissue. J Immunol 135(2 Suppl):755s-s765

24. Hu D, Al-Shalan HAM, Shi Z, Wang P, Wu Y, Nicholls PK et al (2020) Distribution of nerve fibers and nerve-immune cell 
association in mouse spleen revealed by immunofluorescent staining. Sci Rep 10(1):9850

25. Dzierzak E, Bigas A (2018) Blood development: hematopoietic stem cell dependence and independence. Cell Stem Cell 22(5):639-651

26. Kumar BV, Connors TJ, Farber DL (2018) Human T cell development, localization, and function throughout life. Immunity 48(2):202-213

27. Bonne-Annee S, Bush MC, Nutman TB (2019) Differential modulation of human innate lymphoid cell (ILC) subsets by IL-10 and TGF-beta. Sci Rep 9(1):14305

28. Kabata H, Moro K, Koyasu S (2018) The group 2 innate lymphoid cell (ILC2) regulatory network and its underlying mechanisms. Immunol Rev 286(1):37-52

29. Collin M, Ginhoux F (2019) Human dendritic cells. Semin Cell Dev Biol 86:1-2

30. Takenaka MC, Guereschi MG, Basso AS (2017) Neuroimmune interactions: dendritic cell modulation by the sympathetic nervous system. Semin Immunopathol 39(2):165-176

31. Varol C, Mildner A, Jung S (2015) Macrophages: development and tissue specialization. Annu Rev Immunol 33:643-675

32. Davies LC, Taylor PR (2015) Tissue-resident macrophages: then and now. Immunology 144(4):541-548

33. Shapouri-Moghaddam A, Mohammadian S, Vazini H, Taghadosi M, Esmaeili SA, Mardani F et al (2018) Macrophage plasticity, polarization, and function in health and disease. J Cell Physiol 233(9):6425-6440

34. Sun B, Ramberger M, O'Connor KC, Bashford-Rogers RJM, Irani SR (2020) The B cell immunobiology that underlies CNS autoantibody-mediated diseases. Nat Rev Neurol 16(9):481-492

35. Gonzalez-Rey E, Ganea D, Delgado M (2010) Neuropeptides: keeping the balance between pathogen immunity and immune tolerance. Curr Opin Pharmacol 10(4):473-481

36. Krausgruber T, Fortelny N, Fife-Gernedl V, Senekowitsch M, Schuster LC, Lercher A et al (2020) Structural cells are key regulators of organ-specific immune responses. Nature 583(7815):296-302

37. Malcangio M (2019) Role of the immune system in neuropathic pain. Scand J Pain 20(1):33-37

38. Shutov LP, Warwick CA, Shi X, Gnanasekaran A, Shepherd AJ, Mohapatra DP et al (2016) The complement system component C5a Produces thermal hyperalgesia via macrophage-tonociceptor signaling that requires NGF and TRPV1. J Neurosci 36(18):5055-5070

39. Fattori V, Ferraz CR, Rasquel-Oliveira FS, Verri WA Jr (2020) Neuroimmune communication in infection and pain: friends or foes? Immunol Lett 229:32-43

40. Chiu IM, Heesters BA, Ghasemlou N, Von Hehn CA, Zhao F, Tran J et al (2013) Bacteria activate sensory neurons that modulate pain and inflammation. Nature 501(7465):52-57

41. Diogenes A, Ferraz CC, Akopian AN, Henry MA, Hargreaves KM (2011) LPS sensitizes TRPV1 via activation of TLR4 in trigeminal sensory neurons. J Dent Res 90(6):759-764

42. Meseguer V, Alpizar YA, Luis E, Tajada S, Denlinger B, Fajardo $O$ et al (2014) TRPA1 channels mediate acute neurogenic inflammation and pain produced by bacterial endotoxins. Nat Commun $5: 3125$

43. Ferrari LF, Bogen O, Green P, Levine JD (2015) Contribution of Piezo2 to endothelium-dependent pain. Mol Pain 11:65

44. Joseph EK, Green PG, Bogen O, Alvarez P, Levine JD (2013) Vascular endothelial cells mediate mechanical stimulationinduced enhancement of endothelin hyperalgesia via activation of P2X2/3 receptors on nociceptors. J Neurosci 33(7):2849-2859

45. Choi JE, Di Nardo A (2018) Skin neurogenic inflammation. Semin Immunopathol 40(3):249-259
46. Matsuda M, Huh Y, Ji RR (2019) Roles of inflammation, neurogenic inflammation, and neuroinflammation in pain. $\mathrm{J}$ Anesth 33(1):131-139

47. Chu C, Artis D, Chiu IM (2020) Neuro-immune Interactions in the Tissues. Immunity 52(3):464-474

48. Zhang L, Xie W, Zhang J, Shanahan H, Tonello R, Lee SH et al (2021) Key role of CCR2-expressing macrophages in a mouse model of low back pain and radiculopathy. Brain Behav Immun 91:556-567

49. Yu X, Liu H, Hamel KA, Morvan MG, Yu S, Leff J et al (2020) Dorsal root ganglion macrophages contribute to both the initiation and persistence of neuropathic pain. Nat Commun 11(1):264

50. Ceruti S (2020) From astrocytes to satellite glial cells and back: A 25 year-long journey through the purinergic modulation of glial functions in pain and more. Biochem Pharmacol. https:// doi.org/10.1016/j.bcp.2020.114397

51. Lee JH, Kim W (2020) The role of satellite glial cells, astrocytes, and microglia in oxaliplatin-induced neuropathic pain. Biomedicines. https://doi.org/10.3390/biomedicines8090324

52. Zhang H, Li Y, de Carvalho-Barbosa M, Kavelaars A, Heijnen CJ, Albrecht PJ et al (2016) Dorsal root ganglion infiltration by macrophages contributes to paclitaxel chemotherapy-induced peripheral neuropathy. J Pain 17(7):775-786

53. Simeoli R, Montague K, Jones HR, Castaldi L, Chambers D, Kelleher JH et al (2017) Exosomal cargo including microRNA regulates sensory neuron to macrophage communication after nerve trauma. Nat Commun 8(1):1778

54. Chen G, Zhang YQ, Qadri YJ, Serhan CN, Ji RR (2018) Microglia in pain: detrimental and protective roles in pathogenesis and resolution of pain. Neuron 100(6):1292-1311

55. Gong Q, Lin Y, Lu Z, Xiao Z (2020) Microglia-astrocyte cross talk through IL-18/IL-18R signaling modulates migraine-like behavior in experimental models of migraine. Neuroscience 451:207-215

56. Negri L, Maftei D (2018) Targeting the prokineticin system to control chronic pain and inflammation. Curr Med Chem 25(32):3883-3894

57. Gergues M, Nagula V, Bliss SA, Eljarrah A, Ayer S, Gnanavel $\mathrm{N}$ et al (2020) Neuroimmune/hematopoietic axis with distinct regulation by the high-mobility group box 1 in association with tachykinin peptides. J Immunol 204(4):879-891

58. Morelli AE, Sumpter TL, Rojas-Canales DM, Bandyopadhyay M, Chen Z, Tkacheva O et al (2020) Neurokinin-1 receptor signaling is required for efficient $\mathrm{Ca}(2+)$ flux in T-Cell-receptor-activated T cells. Cell Rep 30(10):3448-3465

59. Holzmann B (2013) Antiinflammatory activities of CGRP modulating innate immune responses in health and disease. Curr Protein Pept Sci 14(4):268-274

60. Wallrapp A, Burkett PR, Riesenfeld SJ, Kim SJ, Christian E, Abdulnour RE et al (2019) Calcitonin gene-related peptide negatively regulates alarmin-driven Type 2 innate lymphoid cell responses. Immunity 51(4):709-723

61. Duan JX, Zhou Y, Zhou AY, Guan XX, Liu T, Yang HH et al (2017) Calcitonin gene-related peptide exerts anti-inflammatory property through regulating murine macrophages polarization in vitro. Mol Immunol 91:105-113

62. Maestroni GJM (2020) Adrenergic modulation of hematopoiesis. J Neuroimmune Pharmacol 15(1):82-92

63. Aerts-Kaya F, Ulum B, Mammadova A, Kose S, Aydin G, Korkusuz $P$ et al (2020) Neurological regulation of the bone marrow niche. Adv Exp Med Biol 1212:127-153

64. Hu D, Nicholls PK, Claus M, Wu Y, Shi Z, Greene WK et al (2019) Immunofluorescence characterization of innervation and nerve-immune cell interactions in mouse lymph nodes. Eur J Histochem 63(4):3059 
65. Giudice A, Caraglia M, Marra M, Montella M, Maurea N, Abbruzzese A et al (2010) Circadian rhythms, adrenergic hormones and trafficking of hematopoietic stem cells. Expert Opin Ther Targets 14(5):567-575

66. Maryanovich M, Zahalka AH, Pierce H, Pinho S, Nakahara F, Asada $\mathrm{N}$ et al (2018) Adrenergic nerve degeneration in bone marrow drives aging of the hematopoietic stem cell niche. Nat Med 24(6):782-791

67. Al-Shalan HAM, Hu D, Nicholls PK, Greene WK, Ma B (2019) Immunofluorescent characterization of innervation and nerve-immune cell neighborhood in mouse thymus. Cell Tissue Res 378(2):239-254

68. Leposavic G, Pesic V, Stojic-Vukanic Z, Radojevic K, Arsenovic-Ranin N, Kosec D et al (2010) Age-associated plasticity of alpha1-adrenoceptor-mediated tuning of T-cell development. Exp Gerontol 45(12):918-935

69. Sanders VM (1995) The role of adrenoceptor-mediated signals in the modulation of lymphocyte function. Adv Neuroimmunol 5(3):283-298

70. Dhabhar FS, Malarkey WB, Neri E, McEwen BS (2012) Stress-induced redistribution of immune cells-from barracks to boulevards to battlefields: a tale of three hormonesCurt Richter Award winner. Psychoneuroendocrinology 37(9):1345-1368

71. Nobis CC, Dubeau Laramee G, Kervezee L, Maurice De Sousa D, Labrecque N, Cermakian N (2019) The circadian clock of CD8 $\mathrm{T}$ cells modulates their early response to vaccination and the rhythmicity of related signaling pathways. Proc Natl Acad Sci USA 116(40):20077-20086

72. Beam CA, Wasserfall C, Woodwyk A, Akers M, Rauch H, Blok $\mathrm{T}$ et al (2020) Synchronization of the normal human peripheral immune system: a comprehensive circadian systems immunology analysis. Sci Rep 10(1):672

73. Agac D, Estrada LD, Maples R, Hooper LV, Farrar JD (2018) The beta2-adrenergic receptor controls inflammation by driving rapid IL-10 secretion. Brain Behav Immun 74:176-185

74. Sanders VM (2012) The beta2-adrenergic receptor on $T$ and B lymphocytes: do we understand it yet? Brain Behav Immun 26(2):195-200

75. Sun Z, Hou D, Liu S, Fu W, Wang J, Liang Z (2018) Norepinephrine inhibits the cytotoxicity of NK92MI cells via the beta2adrenoceptor/cAMP/PKA/pCREB signaling pathway. Mol Med Rep 17(6):8530-8535

76. Araujo LP, Maricato JT, Guereschi MG, Takenaka MC, Nascimento VM, de Melo FM et al (2019) The sympathetic nervous system mitigates CNS Autoimmunity via beta2-adrenergic receptor signaling in immune cells. Cell Rep. 28(12):3120-330

77. Tracey KJ (2018) Neurons are the inflammatory problem. Cell 173(5):1066-1068

78. Bassi GS, Kanashiro A, Coimbra NC, Terrando N, Maixner W, Ulloa L (2020) Anatomical and clinical implications of vagal modulation of the spleen. Neurosci Biobehav Rev 112:363-373

79. Murray K, Godinez DR, Brust-Mascher I, Miller EN, Gareau MG, Reardon C (2017) Neuroanatomy of the spleen: mapping the relationship between sympathetic neurons and lymphocytes. PLoS ONE 12(7): 0182416

80. Abe C, Inoue T, Inglis MA, Viar KE, Huang L, Ye H et al (2017) $\mathrm{C} 1$ neurons mediate a stress-induced anti-inflammatory reflex in mice. Nat Neurosci 20(5):700-707

81. Chen WC, Liu YB, Liu WF, Zhou YY, He HF, Lin S (2020) Neuropeptide $\mathrm{Y}$ is an immunomodulatory factor: direct and indirect. Front Immunol 11:580378

82. Bottasso E (2019) Toward the existence of a sympathetic neuroplasticity adaptive mechanism influencing the immune response. A hypothetical view-part I. Front Endocrinol (Lausanne) 10:632
83. Hoover DB, Brown TC, Miller MK, Schweitzer JB, Williams DL (2017) Loss of sympathetic nerves in spleens from patients with end stage sepsis. Front Immunol 8:1712

84. Bottasso E (2019) Toward the existence of a sympathetic neuroplasticity adaptive mechanism influencing the immune response. A hypothetical view-part II. Front Endocrinol (Lausanne) 10:633

85. Vida G, Pena G, Kanashiro A, Thompson-Bonilla Mdel R, Palange D, Deitch EA et al (2011) beta2-Adrenoreceptors of regulatory lymphocytes are essential for vagal neuromodulation of the innate immune system. FASEB J 25(12):4476-4485

86. Fujii T, Mashimo M, Moriwaki Y, Misawa H, Ono S, Horiguchi $K$ et al (2017) Physiological functions of the cholinergic system in immune cells. J Pharmacol Sci 134(1):1-21

87. Hoover DB (2017) Cholinergic modulation of the immune system presents new approaches for treating inflammation. Pharmacol Ther 179:1-16

88. Huang Y, Zhao C, Su X (2019) Neuroimmune regulation of lung infection and inflammation. QJM 112(7):483-487

89. Benarroch EE (2019) Autonomic nervous system and neuroimmune interactions: new insights and clinical implications. Neurology 92(8):377-385

90. Matteoli G, Gomez-Pinilla PJ, Nemethova A, Di Giovangiulio M, Cailotto C, van Bree SH et al (2014) A distinct vagal antiinflammatory pathway modulates intestinal muscularis resident macrophages independent of the spleen. Gut 63(6):938-948

91. Veiga-Fernandes H, Pachnis V (2017) Neuroimmune regulation during intestinal development and homeostasis. Nat Immunol 18(2):116-122

92. Kulkarni S, Ganz J, Bayrer J, Becker L, Bogunovic M, Rao M (2018) Advances in enteric neurobiology: the "brain" in the gut in health and disease. J Neurosci 38(44):9346-9354

93. Varricchi G, Pecoraro A, Loffredo S, Poto R, Rivellese F, Genovese A et al (2019) Heterogeneity of human mast cells with respect to MRGPRX2 receptor expression and function. Front Cell Neurosci 13:299

94. Lou H, Lu J, Choi EB, Oh MH, Jeong M, Barmettler S et al (2017) Expression of IL-22 in the skin causes Th2-biased immunity, epidermal barrier dysfunction, and pruritus via stimulating epithelial Th2 cytokines and the GRP pathway. J Immunol 198(7):2543-2555

95. Azimi E, Xia J, Lerner EA (2016) Peripheral mechanisms of itch Curr Probl Dermatol 50:18-23

96. Hou Q, Barr T, Gee L, Vickers J, Wymer J, Borsani E et al (2011) Keratinocyte expression of calcitonin gene-related peptide beta: implications for neuropathic and inflammatory pain mechanisms. Pain 152(9):2036-2051

97. Wang A, Bai Y (2020) Dendritic cells: the driver of psoriasis. J Dermatol 47(2):104-113

98. Cohen JA, Edwards TN, Liu AW, Hirai T, Jones MR, Wu J et al (2019) Cutaneous TRPV1(+) neurons trigger protective innate Type 17 anticipatory immunity. Cell 178(4):919-932

99. Cooper MS, Clark VP (2013) Neuroinflammation, neuroautoimmunity, and the co-morbidities of complex regional pain syndrome. J Neuroimmune Pharmacol 8(3):452-469

100. Tajerian M, Clark JD (2016) New concepts in complex regional pain syndrome. Hand Clin 32(1):41-49

101. Stanton-Hicks MD (2019) CRPS: what's in a name? Taxonomy, epidemiology, neurologic, immune and autoimmune considerations. Reg Anesth Pain Med 44(3):376-387

102. De Logu F, De Pra SD, de David Antoniazzi CT, Kudsi SQ, Ferro PR, Landini L et al (2020) Macrophages and Schwann cell TRPA1 mediate chronic allodynia in a mouse model of complex regional pain syndrome type I. Brain Behav Immun 88:535-546

103. Li W, Shi X, Wang L, Guo T, Wei T, Cheng K et al (2013) Epidermal adrenergic signaling contributes to inflammation and pain 
sensitization in a rat model of complex regional pain syndrome. Pain 154(8):1224-1236

104. Sui P, Wiesner DL, Xu J, Zhang Y, Lee J, Van Dyken S et al (2018) Pulmonary neuroendocrine cells amplify allergic asthma responses. Science 360:6393

105. Nagashima H, Mahlakoiv T, Shih HY, Davis FP, Meylan F, Huang Y et al (2019) Neuropeptide CGRP limits Group 2 innate lymphoid cell responses and constrains type 2 inflammation. Immunity 51(4):682-695

106. Feng X, Li L, Feng J, He W, Li N, Shi T et al (2020) Vagalalpha7nAChR signalling attenuates allergic asthma responses and facilitates asthma tolerance by regulating inflammatory group 2 innate lymphoid cells. Immunol Cell Biol. https://doi. org/10.1111/imcb. 12400

107. Cardoso V, Chesne J, Ribeiro H, Garcia-Cassani B, Carvalho T, Bouchery T et al (2017) Neuronal regulation of type 2 innate lymphoid cells via neuromedin U. Nature 549(7671):277-281

108. Khalil M, Zhang Z, Engel MA (2019) Neuro-immune networks in gastrointestinal disorders. Visc Med 35(1):52-60

109. Kulkarni S, Kurapati S, Bogunovic M (2020) Neuro-innate immune interactions in gut mucosal immunity. Curr Opin Immunol 68:64-71
110. Albert-Bayo M, Paracuellos I, Gonzalez-Castro AM, RodriguezUrrutia A, Rodriguez-Lagunas MJ, Alonso-Cotoner $\mathrm{C}$ et al (2019) Intestinal mucosal mast cells: key modulators of barrier function and homeostasis. Cells 8(2):135

111. Verheijden S, Boeckxstaens GE (2018) Neuroimmune interaction and the regulation of intestinal immune homeostasis. Am J Physiol Gastrointest Liver Physiol 314(1):G75-G80

112. Willemze RA, Welting $O$, van Hamersveld $P$, Verseijden $C$, Nijhuis LE, Hilbers FW et al (2019) Loss of intestinal sympathetic innervation elicits an innate immune driven colitis. Mol Med 25(1): 1

113. Song J, Wang T, Zhang X, Li B, Zhu C, Zhang S (2020) Upregulation of gastric norepinephrine with beta-adrenoceptors and gastric dysmotility in a rat model of functional dyspepsia. Physiol Res 69(1):135-143

114. Grubisic V, McClain JL, Fried DE, Grants I, Rajasekhar P, Csizmadia E et al (2020) Enteric glia modulate macrophage phenotype and visceral sensitivity following inflammation. Cell Rep 32(10): 108100 